2. Brown SL, Gropler RJ, Harris KM. Distinguishing left ventricular aneurysm from pseudoaneurysm. Chest. 1997;111:1403-9.

3. Yeo TC, Malouf JF, Oh JK, Seward JB. Clinical profile and outcome in 52 patients with cardiac pseudoaneurysm. Ann Intern Med. 1998;128: 299-305.
4. Pretre R, Linka A, Jenni R, Turina MI. Surgical treatment of acquired left ventricular pseudoaneurysms. Ann Thorac Surg. 2000;70:553-7.

5. Moreno R, Gordillo E, Zamorano J, Almeria C, Garcia-Rubira JC, Fernandez-Ortiz A, et al. Long term outcome of patients with postinfarction left ventricular pseudoaneurysm. Heart. 2003;89:1144-6.

\title{
Atrial septal mass: Transesophageal echocardiographic assessment
}

\author{
Giulio Rizzoli, MD, ${ }^{a}$ Tomaso Bottio, MD, PhD, ${ }^{a}$ Demetrio Pittarello, MD, ${ }^{a}$ Massimo Napodano, MD, ${ }^{c}$ Gaetano Thiene, MD, \\ and Cristina Basso, MD, $\mathrm{PhD},{ }^{\mathrm{b}}$ Padua, Italy
}

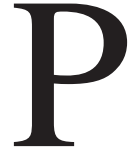

rimary tumors are very rare, with the incidence in necroscopy series being between $0.0017 \%$ and $0.28 \%{ }^{1}$ Angiomas account for less than $5 \%$ of all these. Today, few reports discussing the clinical manifestation and pathologic appearance of cardiac angiomas have been published worldwide in the literature, and very few articles report surgical angioma excision. ${ }^{2}$

The angioma is a histologically benign tumor without infiltrative propensity, and it is mostly described in the subendocardial layers of the right atrium in adult female patients.

Although these are incidental findings in the majority of cases because they are asymptomatic, such tumors might be associated with a wide range of atypical and different symptoms that are related to the cardiac chambers involved or to the specific cardiac site of origin.

At gross examination, this tumor appears like a circumscribed mass in the subendocardial layer. Echocardiographic imaging techniques, either transthoracic or transesophageal, allow a differential diagnosis, whereas cardiac catheterization appears to be unnecessary. We report the case of a symptomatic patient undergoing successful surgical intervention for an interatrial septal angioma detected by means of 2-dimensional echocardiography.

\section{Clinical Summary}

A 62-year-old woman with effort-induced dyspnea was referred to us with transesophageal echocardiography-based diagnosis of an atrial septal mass. Physical examination was unremarkable without the appearance of a cardiac murmur. A 12-lead electrocardiogram showed normal sinus rhythm, without evidence of pathologic waves. The transesophageal echocardiogram showed a lobulated,

\footnotetext{
From the Institute of Cardiovascular Surgery, ${ }^{\mathrm{a}}$ Cardiology, ${ }^{\mathrm{b}}$ and Pathology, University of Padua Medical School, Padua, Italy.

Received for publication March 3, 2004; accepted for publication March 22, 2004.

Address for reprints: Cristina Basso, $\mathrm{MD}, \mathrm{PhD}$, Istituto di Anatomia Patologica, Via A. Gabelli, 61, 35121 Padova, Italy (E-mail: cristina.basso@ unipd.it).

J Thorac Cardiovasc Surg 2004;128:767-9

$0022-5223 / \$ 30.00$

Copyright (C) 2004 by The American Association for Thoracic Surgery

doi:10.1016/j.jtcvs.2004.03.053
}

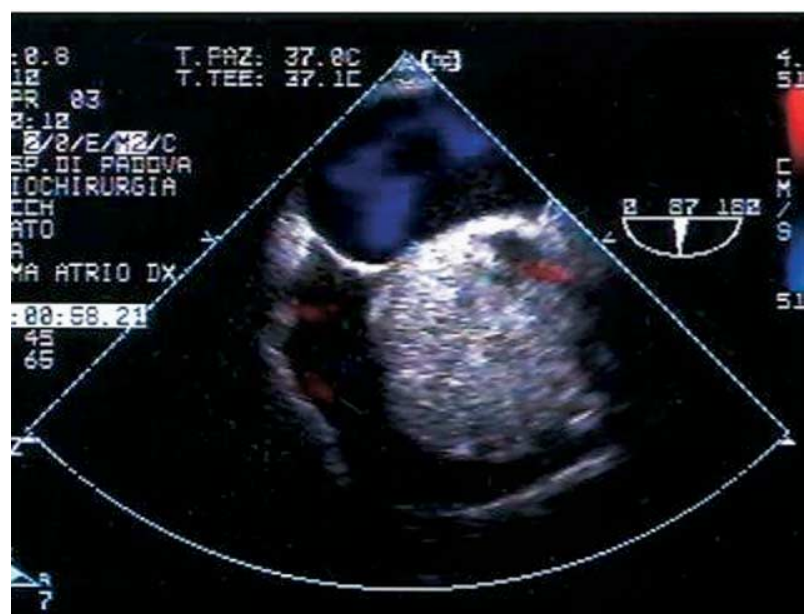

Figure 1. Transesophageal echocardiography shows an interatrial septal cystic mass that interferes with right blood flow by protruding into the right atrium and that is limited in its extension by the endocardial layers. At Doppler examination, an afferent coronary branch is discovered. The left atrium is at the top of the screen.

fixed mass $(6.4 \mathrm{~cm}$ in diameter), which was related to the atrial septum (Figure 1) and was severely restricting the superior vena caval inflow. The tumor expanded in the right atrium, which was also enlarged. Within the tumor mass, a cyst had been discovered with an afferent arterial vessel by means of color Doppler analysis. The right ventricle and left cardiac chambers were within normal values for dimension and function. Tricuspid leaflet motion was not altered, and the valve was continent. Cardiac catheterization was performed showing the tumor surrounded by a laminar stream of caval blood and normal coronary arteries. A faint afferent artery originating from the right coronary artery and a faint contrast uptake within the atrial septum could be recognized (Figure 2).

The patient underwent surgical intervention with cardiopulmonary bypass during moderate hypothermia and retrograde cardioplegic arrest. Through a transatrial approach, the bulky atrial septum was carefully incised, and a large spherical mass of $3 \mathrm{~cm}$ in diameter was enucleated from the septal wall. The large cavity at the base of the atrial septum was then obliterated by suturing. Tricuspid valve and mitral valve leaflets appeared normal and 


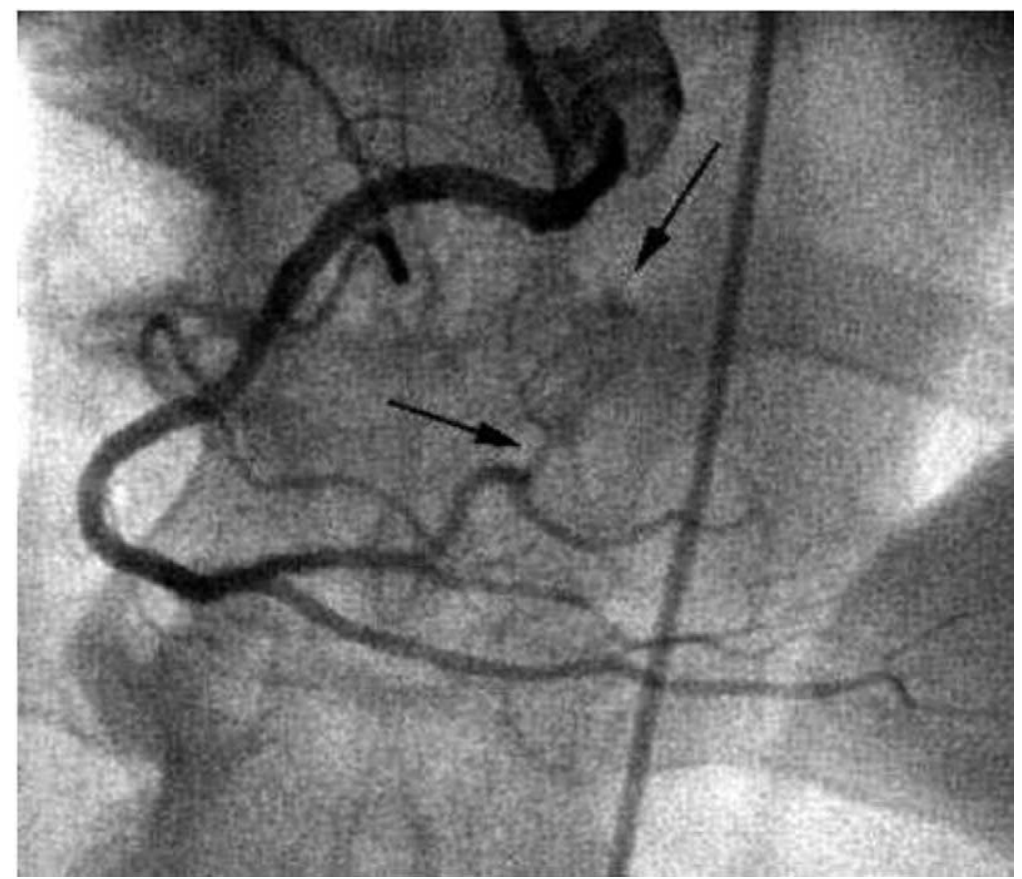

Figure 2. Catheter angiography, performed to exclude coronary lesions in view of the surgical excision, confirmed the presence of a coronary artery supply to the mass.
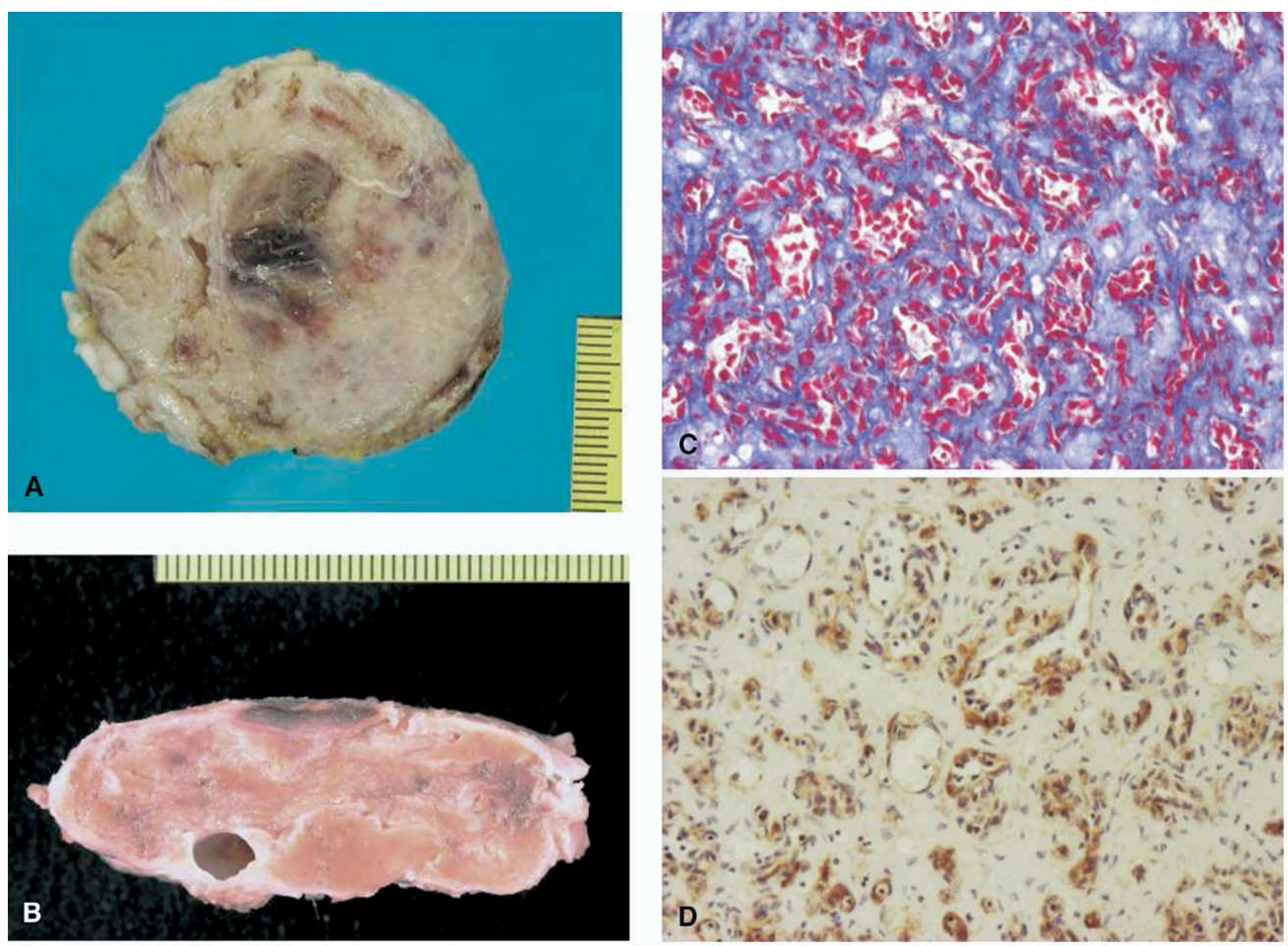

Figure 3. A and B, At gross examination, the excised mass appears round, solid, and flattened, $5 \times 5 \times 2 \mathrm{~cm}$ in size, with cystic spaces at the transverse cut. C and D, Histology and immunohistochemistry reveal multiple vascular spaces with endothelial lining. (Original magnification $25 \times$; trichrome and CD31 staining.) 
continent, without evidence of residual tumor. An intraoperative transesophageal echocardiogram showed normal valve function with no residual incompetence. The patient was weaned off cardiopulmonary bypass quickly. Gross and histologic examination led to a final diagnosis of atrial septal angioma (Figure 3).

\section{Discussion}

Despite being histologically benign, cardiac angiomas, depending on the dimension and the cardiac site localization, are potentially life-threatening.

Van der Hanwaert ${ }^{3}$ reported 2 cases of angioma localized in the interventricular septum causing atrioventricular block, which was complete in one patient, who died suddenly.

Therefore even when the extent of the lesion is limited, these tumors are likely to be considered for surgical excision. If they grow, involving a large amount of atrial or ventricular tissue, complete tumor resection could be impossible.

The diagnosis of these intracavitary masses is made typically by means of echocardiography, either transthoracic or transesophageal. Transesophageal echocardiography has been proved to be superior to the transthoracic echocardiography in establishing a differential diagnosis with the other intracavitary cardiac tumors, such as myxoma, rhabdomyoma, fibroma, and teratoma. The subendocardial position of cardiac angiomas permitted us to exclude the myxoma, which typically is an intracavitary tumor attached to the endocardium of interatrial septum. The angioma cyst appearance and the coronary afferent vessel identified at Doppler screening and angiography permitted us to distinguish it from rhabdomy- oma and fibroid tumor, which are solid masses. The hypothesis of teratomas, which are multicystic, calcified, and rare intracardiac tumors, can be excluded in the absence of calcification.

A definitive response rests on the histologic examination of the excised mass. In this case a complete surgical excision was possible, enucleating the mass from within the septal wall and the atrial septum. On the other hand, when limits between the tumor and the cardiac muscle, mostly interventricular septum, are less recognizable, the operation might be destructive and not resoluble. The afferent arteries were not identified, and therefore they were divided with other adhesions during the mass enucleation. The atrial septum can be either removed and replaced with a pericardial patch or, as in our case, the cavity can be obliterated with separate stitches to avoid filling of the residual cavity by coronary flow.

This experience highlights the value of transesophageal echocardiography in the differential diagnosis of primary cardiac tumors, allowing the recognition even of very rare cardiac tumors and also showing their precise location and topographic relationship with other cardiac structures.

\section{References}

1. Burke A, Virmani R. Tumors of the heart great vessels. In: Atlas of tumor pathology, fascicle 16, series III. Washington, DC: Armed Forces Institute of Pathology; 1996.

2. Ilbawi M, DeLeon S, Riggs T, Hernandez R. Primary vascular tumors of the heart in infancy. Chest. 1982;81:511-2.

3. Van der Hanwaert LG. Cardiac tumors in infancy and childhood. $\mathrm{Br}$ Heart J. 1971;33:125-32. 